\title{
EVALUACIÓN DE LA DENSIDAD DE TRAMPAS DE FEROMONA EN LA CAPTURA DE LA POLILLA CENTROAMERICANA DE LA PAPA (Tecia solanivora Povolny)
}

\author{
José de J. Alvarado*;Eduardo Ortega C. **;José Acevedo C.*
}

\section{RESUMEN}

La polilla centroamericana de la papa Tecia solanivora Povolny ocasiona daños a la producción hasta el $100 \%$ de los tubérculos en las zonas afectadas. Se estudió diferentes densidades (10, 20, 30 y 40 trampas por ha) utilizando feromona sexual en trampas de agua diseñadas para la captura de adultos. La actividad se ejecutó cerca de la localidad de Mucuchies, a 3100 msnm en un área que representa el $41 \%$ de la superficie productora de papa del estado Mérida, Venezuela. Se consideraron los promedios de captura por trampa cada siete días, relacionándolos con las condiciones climáticas, especialmente precipitación, observándose una relación inversa. El análisis crítico de los resultados periódicos de capturas, distribución y número de trampas por unidad de superficie indican que las densidades de 10 y 20 trampas/ha son las mejores bajo las condiciones estudiadas, con poca significancia entre una y otra densidad. Se estima que desde el punto de vista económico y práctico 10 trampas/ha es lo más conveniente.

Palabras claves adicionales: papa, confusión de machos.

Aceptado para publicación: abril 15,1995

* Ing. Agr ; Br. Agrop. FONAIAP - Centro de Investigaciones Agropecuarias - Estado Mérida. Apartado Aéreo 425, Mérida 5101 Venezuela.

** Ing. Agr. M.S. FONAIAP - Centro de Investigaciones Agropecuarias Estado Monagas. Apartado Aéreo 184-A. Maturin, Venezuela. 


\section{SUMMARY}

\section{EVALUATION OF THE DENSITY OF SEXUAL PHEROMONE TRAPS IN THE CAPTURE OF THE CENTRAL AMERICAN POTATO TUBER MOTH (Tecia solanivora Povolny)}

The potato tuber moth (Tecia solanivora Povolny) can cause up to $100 \%$ damage of the tuber production in endemic zones. Four sexual pheromone trap densities (10, 20, 30 and 40 traps/ha) were tested in a field located near Mucuchics (3100 masl), Venezuela. Male capture was evaluated every seven days, relating them with environmental conditions; relationship with rain was inverse. Results indicated that 10 and 20 traps/ha were more effective in capturing males; there were minor significant differences among these two densities. From the economical and practical points of view, it is recommended to use 10 traps/ha.

Additional index words: $\quad$ potato, male confusion.

El cultivo de la papa Solanum tuberosum L. constituye un rubro de gran importancia económica en Venezuela por su superficie sembrada y volumen de producción y es realizado principalmente por pequeños productores (7).

La papa es atacada por diversos insectos-plagas, cuya importancia depende de la región ecológica de siembra, y algunos de ellos tienen importancia económica en todas las áreas paperas del país (18).

La polilla centroamericana de la papa (Tecia solanivora Povolny) fue introducida en 1983 con una importación de semilla de la variedad Atzimba al estado Tachira (16) alcanzando en poco tiempo una distribución amplia y muy buena adaptabilidad en varias localidades de ese estado (17). De allí se diseminó a los estados Mérida y Trujillo (5) y su presencia ha sido señalada en 1987 en el estado Lara (15) y en mercados al detal de papa para consumo del estado Aragua (Notz citado por Torres (19). De Venezuela se diseminó a Colombia (4).

El daño económico lo causa la larva, la cual una vez que eclosiona el huevo se orienta hacia el tubérculo, raspa su superficie y penetra debajo de la epidermis y 
luego barrena más profundamente hasta formar galerías dentro del tubérculo (12, 19). El ataque puede ocurrir tanto en campo como en almacén, constituyendo en este último caso, un verdadero problema para los agricultores del área andina de Venezuela. Las pérdidas oscilan entre 25-50\% en los países centroamericanos, (14) y puede llegar al 100\% si no se ejerce ninguna acción de control (3).

En la estrategia de manejo integrado de esta plaga en Venezuela se han incluido estudios bioecológicos, prácticas culturales y control biológico, etológico, químico y por variedades resistentes (10).

La feromona sexual de Tecia solanivora fue aislada en 1980 (6) y es utilizada con éxito como atrayente de machos adultos y en estudios de niveles de dinámica poblacional $(1,2,17)$.

El control de machos de polillas con feromonas es de bajo costo, requiere poca inversión en mano de obra, no daña el equilibrio ecológico y biológico del medio y permite la acción de los enemigos naturales de la plaga (9).

Dada las diferencias de poblaciones en diversas localidades, la influencia del cultivo de papa y de las condiciones climáticas, el objetivo de este trabajo fue determinar el número de trampas provistas con las feromona sexual, a distribuir por unidad de superficie para lograr una mayor captura.

\section{MATERIALES Y MÉTODOS}

Ubicación: La actividad se ejecutó en un área localizada en la cuenca del río Chama, que representa el $41 \%$ de la producción del estado de Mérida (8). Esta se estableció cercana a la población de Mucuchíes, a 3100 msnm en las fincas de productores "La Mesa" y "Mimuri" con siembras comerciales de las variedades de papa Andinita y Monserrate, ambas de ciclo largo (4.5-5.0 meses).

Las trampas fueron colocadas en el campo antes de la emergencia de las plantas y permanecieron en el mismo por algunas semanas más, después de que se realizó la cosecha a los 160 días.

Las trampas son de plástico color amarillo, diseñadas para captura masiva de polillas adultas y se llenaron con agua hasta $2 \mathrm{~cm}$ por debajo del borde del recipiente, agregándole una gotas de Citowett pluss para disminuir la tensión superficial de la misma y facilitar la muerte, el rápido hundimiento y el contaje evitando el aglutinamiento de los insectos capturados. El dedal de goma impregnado con la feromona sexual sintética fue colocado encima a $2 \mathrm{~cm}$ de superficie del agua, mediante un gancho de alambre colgado de la cubierta de la trampa (Figura 1). 


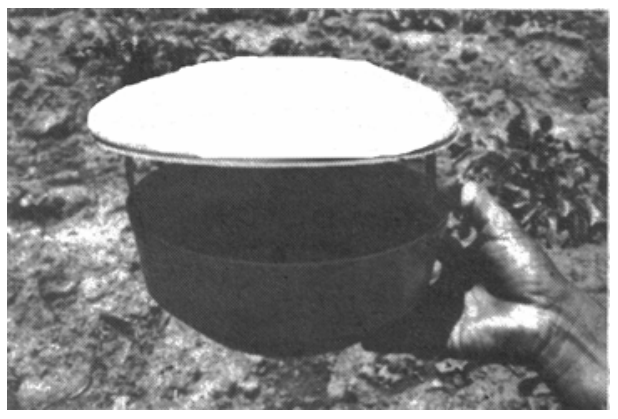

Figura 1.

Densidad y colocación de las trampas: La distribución de las trampas por cada hectárea del cultivo se hizo de acuerdo al croquis presentado en la Figura 2. Considerando las cuatro densidades, el espaciamiento entre trampas quedó en el campo de la manera siguiente:

\section{Para 10 trampas/ha}

- Cuatro alrededor de los bordes, una por cada lado, distanciadas a 50m de las esquinas.

- Seis dentro de la siembra, dispuestas en tres hileras de dos trampas, quedando las dos de la hilera central separadas a $25 \mathrm{~m}$ y las dos de las hileras laterales a $50 \mathrm{~m}$ entre ellas y a $25 \mathrm{~m}$ de los bordes.

Para 20 trampas/ha.

- Ocho alrededor de los bordes, dos de ellas por cada lado, distanciadas entre si $50 \mathrm{~m}$ y separadas de las esquinas a $25 \mathrm{~m}$.

- Doce dentro de la siembra, dispuestas en tres hileras de cuatro trampas, dejando una separación entre ellas y bordes de la siembra de $20 \mathrm{~m}$. En el otro sentido de la hilera las trampas quedan separadas 25 m entre sí y el borde.

\section{Para 30 trampas / ha}

- Doce al contorno, tres por cada lado, distanciadas entre ellas y las esquinas $25 \mathrm{~m}$.

- Dieciocho dentro del cultivo, dispuestas en tres hileras de seis, dejando una separación entre trampas y bordes dentro de la hilera de aproximadamente $14.28 \mathrm{~m}$ en sentido contrario a la hilera las mismas quedan separadas $20 \mathrm{~m}$ entre sí y el borde.

\section{Para 40 trampas / ha}

- Dieciséis alrededor de los bordes, cuatro por lado, separadas entre sí y de las esquinas $20 \mathrm{~m}$.

- Veinticuatro dentro del cultivo, distribuidas en cuatro hileras de las seis trampas, separadas dentro de la hilera $14.28 \mathrm{~m}$, aproximadamente entre sí y el borde, quedando en el otro sentido de las hileras separadas 20 m entre sí y el borde correspondiente. 
Las trampas colocadas en los bordes capturan los machos que emigran o se mueven dentro del cultivo, mientras que las internas capturan los insectos que están dentro del mismo. En el campo inicialmente las trampas fueron colocadas directamente sobre el nivel del suelo y una vez desarrollado el cultivo se levantaron de la superficie a una altura de $60 \mathrm{~cm}$ mediante soportes especiales de maderas para facilitar la dispersión de olor de la feromona.

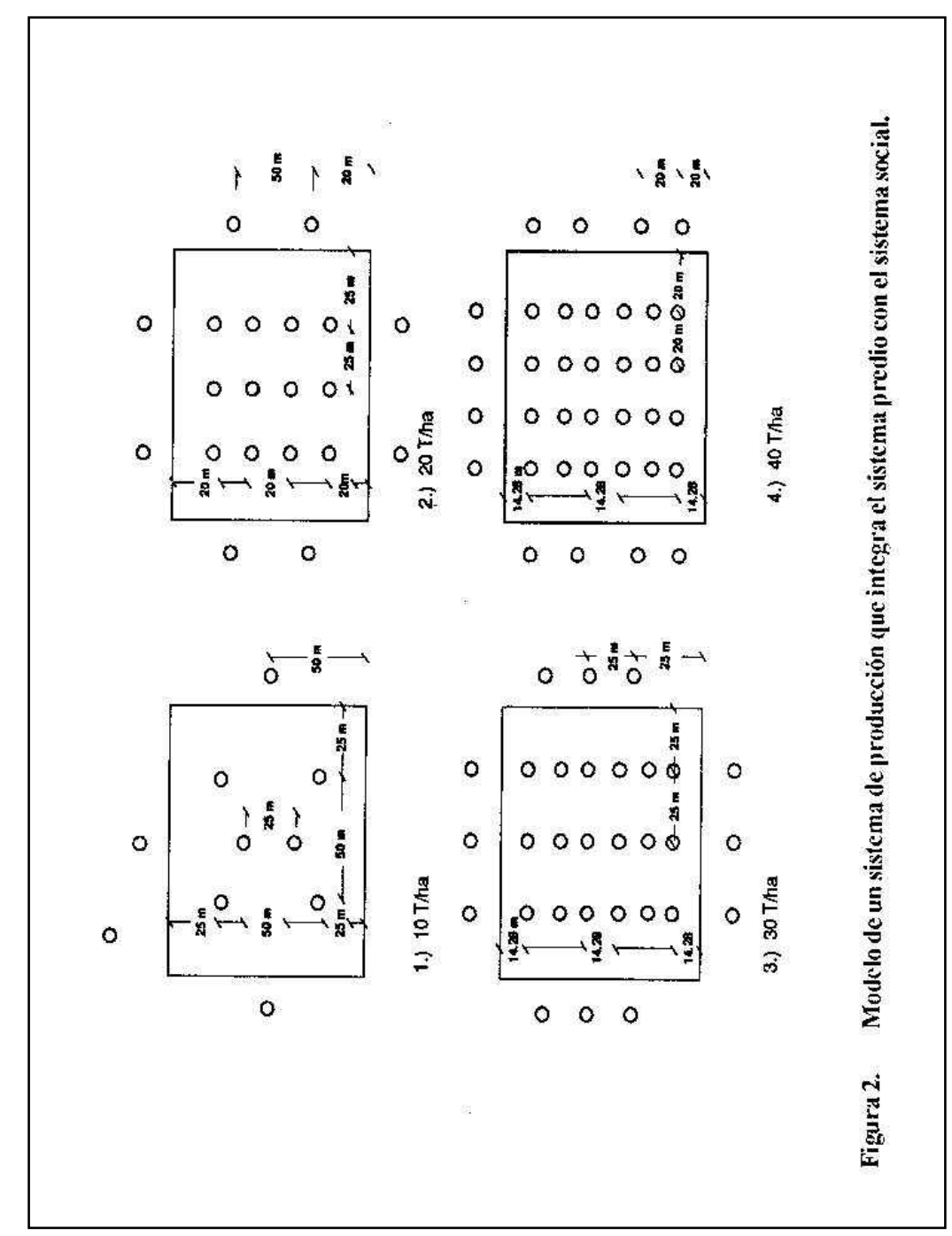


Colecta de la información: Los adultos capturados se contaron manualmente y se anotó el número obtenido en una planilla diseñada para tal fin. Las trampas de agua eran revisadas y limpiadas por contaje cada siete dias.

Se realizaron 29, 34, 26 y 19 contajes para la densidades de 10,20, 30 y 40 T / ha en períodos de captura en 1989 del 13-04 al 26-10 (193 días) del 14-03 al 31-10 (227 días), del 11-04 al 02-11 (171 días) y del 29-05 al 02-10 (123 días), respectivamente.

Durante el período de evaluación se registró la información (Tabla 1) referente a precipitación, temperatura y humedad relativa de la unidad climatológica del Campo Experimental de Mucuchíes de la Estación Experimental Mérida, ubicado en el área de influencia del experimento.

Tabla 1. Precipitación, temperatura y humedad relativa registrada durante la evaluación de cuatro densidades de trampas con feromona sexual.

\begin{tabular}{llllll}
\hline Mes & $\mathrm{P}(\mathrm{mm})$ & $\begin{array}{l}\text { T.Max. } \\
{ }^{\circ} \mathrm{C}\end{array}$ & $\begin{array}{l}\text { T.Min. } \\
{ }^{\circ} \mathrm{C}\end{array}$ & $\begin{array}{l}\text { T.Med. } \\
{ }^{\circ} \mathrm{C}\end{array}$ & H.R.\% \\
\hline Mar & 36.70 & 16.17 & 5.00 & 10.60 & 78 \\
Abr & 7.10 & 18.06 & 5.80 & 11.93 & 66 \\
May & 91.50 & 16.60 & 6.60 & 11.50 & 75 \\
Jun & 80.10 & 15.10 & 6.20 & 11.60 & 70 \\
Jul & 76.70 & 15.15 & 6.18 & 10.70 & 74 \\
Ago & 77.50 & 16.20 & 6.50 & 11.35 & 75 \\
Set & 125.90 & 16.20 & 6.20 & 11.20 & 72 \\
Oct & 76.70 & 16.45 & 6.33 & 11.39 & 70 \\
Nov & 1.50 & 16.48 & 6.09 & 11.28 & 67 \\
& & & & & \\
\hline Total & $\begin{array}{l}\text { Promedio } \\
\end{array}$ 573.70 & 16.23 & 6.10 & 11.17 & 71.88 \\
\hline
\end{tabular}

\section{RESULTADOS Y DISCUSIÓN}

Durante el período total de evaluación (08-03 a 31-10) el número total de capturas fue de 19 784, 82 222, 36918 y 15432 individuos en las densidades de 10, 20, 30 y 40 trampas/ ha respectivamente, con un promedio semanal portrampa de 68.2, 16.9, 47.3 y 20.3 (Tabla 2). 
Tabla 2. Fluctuación poblacional de machos adultos de Tecla solanivora (Povolny) capturados en cuatro densidades de trampas de agua con feromona sexual.

\begin{tabular}{|lllll|}
\hline $\begin{array}{l}\text { Machos adultos } \\
\text { Capturados }\end{array}$ & \multicolumn{4}{c|}{$\begin{array}{c}\text { Densidad } \\
\text { Número de trampas por ha. }\end{array}$} \\
\cline { 2 - 5 } & 10 & 20 & 30 & 40 \\
\hline $\begin{array}{l}\text { Total } \\
\text { Promedio trampas }\end{array}$ & 19.784 & 82.222 & 36.918 & 15.432 \\
$\begin{array}{l}\text { Semana } \\
\text { Día }\end{array}$ & 68.2 & 16.9 & 47.3 & 20.3 \\
Máxima semana & 9.7 & 2.4 & 6.8 & 2.9 \\
Mínimo semana & 419.0 & 417.2 & 170.6 & 34.9 \\
& 5.3 & 4.4 & 17.9 & 4.9 \\
\hline
\end{tabular}

Las máximas capturas se realizaron durante el desarrollo del cultivo en las primeras seis semanas de evaluación cuando el régimen de precipitación fue de sólo $40.7 \mathrm{~mm}$; en estas circunstancias los valores máximos fueron de 471, 419, 171 y 35 adultos en la distribución de 10, 20, 30 y 40 T / ha. A partir de esa lectura los promedios de capturas semanales fueron disminuyendo, observándose muy pocas variaciones para las diferentes densidades hacia la época de maduración de la plantación y máximo desarrollo de los tubérculos (Figura 3).

Durante el lapso posterior a la cosecha se observó que la población insectil, reflejada por las capturas promedio de adultos se mantienen relativamente baja y casi constante en las diferentes densidades, registrándose valores mínimos de capturas semanales. En este caso la precipitación del lapso 25-04 al 31 -10 fue de $528.4 \mathrm{~mm}$.

Las mayores capturas indican una mayor población activa del insecto en el primer período del cultivo y el efecto de relación inversa de la población capturada y el régimen pluviométrico) fue observado también por Rocha (11).

No se observaron diferencias de capturas entre las dos variedades, debido probablemente a las características similares entre ellas dado que Andinita tiene como progenitura a Monserrate (20).

Los datos de captura seleccionados y agrupados para el período juniosetiembre para las cuatro densidades se presentan en la Tabla 3, donde el mayor número de machos adultos se registro con $20 \mathrm{~T} /$ ha el análisis estadístico de los datos transformados mostró diferencias significativas para las densidades al nivel del $10 \sim$ y no para los meses de evaluación. La comparación de los promedios con sus respectivas desviaciones estándar (Tabla 3), indican que estas tienden a disminuir en la medida que se incrementa la densidad de trampas, demostrando que la distribución de un número grande de ellas induce a una menor variabilidad en la captura entre tamaño y trampa para esta especie de polilla. 


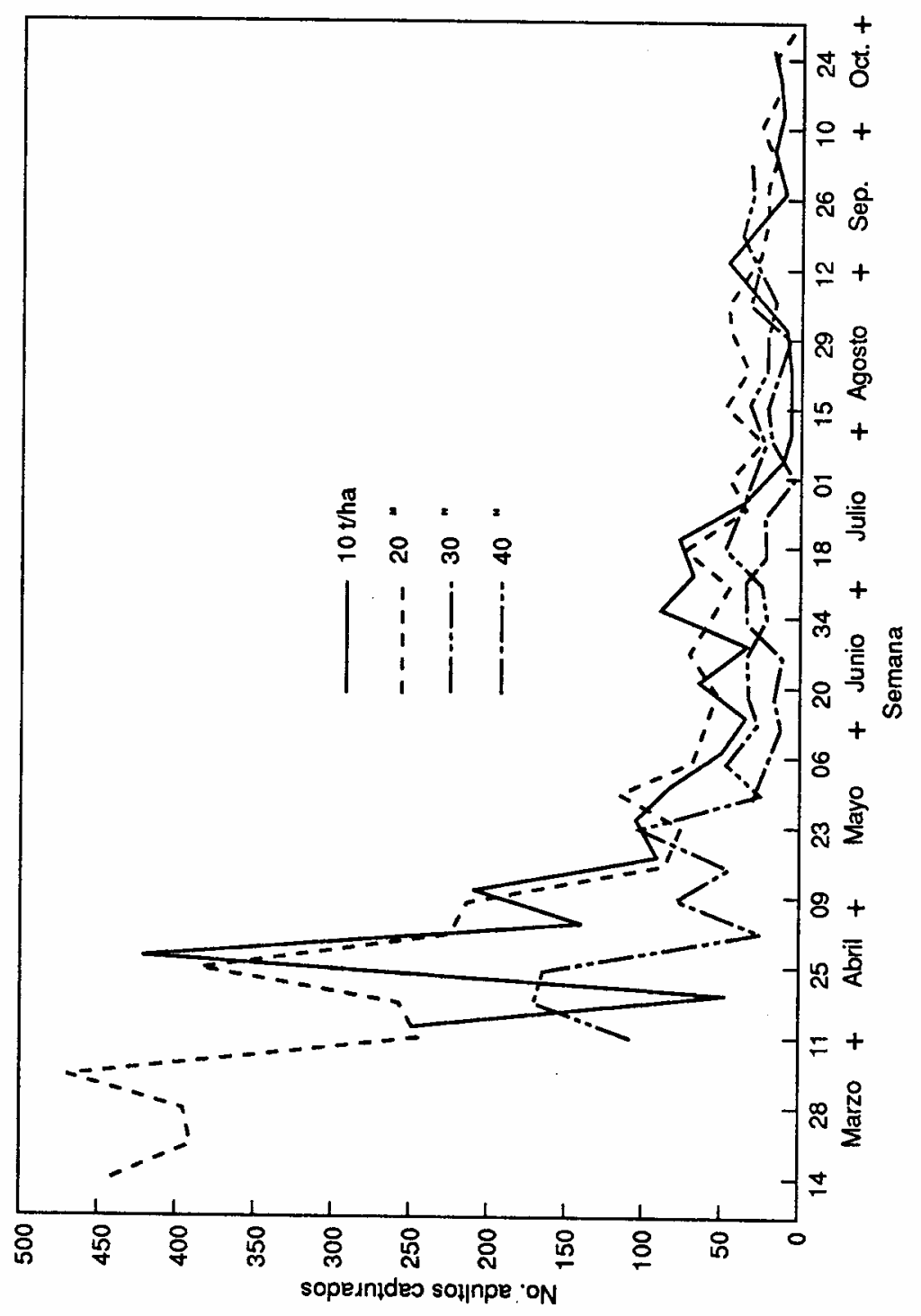

Figura 3. Fluctuación poblacional de adultos machos de Tecia solanivora (Povolny), capturados en campo en densidades de 10, 20, 30 y 40 trampas de agua con feromona. 
Tabla 3. Total de resultados de Tecia solanivora Povolny capturados mensualmente en trampas de agua con feromona sexual distribuidas en campo a densidades de 10, 20, 30 trampas/ha.

\begin{tabular}{|c|c|c|c|c|}
\hline \multirow[b]{2}{*}{ Meses } & \multicolumn{4}{|c|}{ Número de trampas/ha } \\
\hline & 10 & 20 & 30 & 40 \\
\hline Junio & 182.0 & 252.8 & 135.8 & 67.7 \\
\hline Julio & 274.4 & 245.1 & 155.0 & 113.8 \\
\hline Agosto & 27.4 & 134.0 & 92.7 & 55.4 \\
\hline Setiembre & 99.3 & 114.2 & 107.1 & 101.6 \\
\hline $\mathrm{X}$ & 145.78 & 188.78 & 122.65 & 84.63 \\
\hline S.D. & 106.50 & 70.54 & 28.04 & 27.57 \\
\hline
\end{tabular}

Los medios de captura de machos por mes y por trampa se ajustan perfectamente a una función polinomial de tercer orden, cuya ecuación fue: $\mathrm{Y}=-143.59+45.83 \mathrm{X}-1.918 \mathrm{X} 2+2.287 \mathrm{e}-2 \mathrm{X} 3$ con $1 \mathrm{R} 2=1.00$ de alto valor predictivo. (Figura 4).

En la prueba de rango múltiple de Duncan se encontró que el tratamiento de $40 \mathrm{~T}$ / ha capturó un número de machos estadísticamente diferente a los otros tratamientos estudiados (Figura 5).

Las capturas menores en las distribuciones de 30 y $40 \mathrm{~T}$ / ha podrían explicarse por el efecto de confusión del insecto (9), ya que por haber una mayor dispersión del atrayente sexual por unidad de superficie, debido al mayor número de trampas, el poder individual de atracción de estas es menor y en consecuencia, menor el número de insectos capturados por trampa.

Desde el punto de vista económico y práctico la distribución de 10 T / ha es más conveniente por la menor inversión que se hace en su adquisición y la utilización de menos jornales para el buen servicio de mantenimiento de los mismas en el campo. En Costa Rica, para áreas paperas menores a 2500 m.s.n.m se ha recomendado la colocación de 12-16 T/ ha $(13,14)$.

\section{AGRADECIMIENTO}

Los autores expresan su agradecimiento a los Drs. K. V. Raman y L. Valencia, del Centro Internacional de la Papa por el suministro de las muestras de feromonas y por las sugerencias y correcciones al manuscrito original, respectivamente. Al Programa Andino Cooperativo de Investigaciones en Papa (PRACIPA) por el financiamiento para la ejecución de este trabajo. 


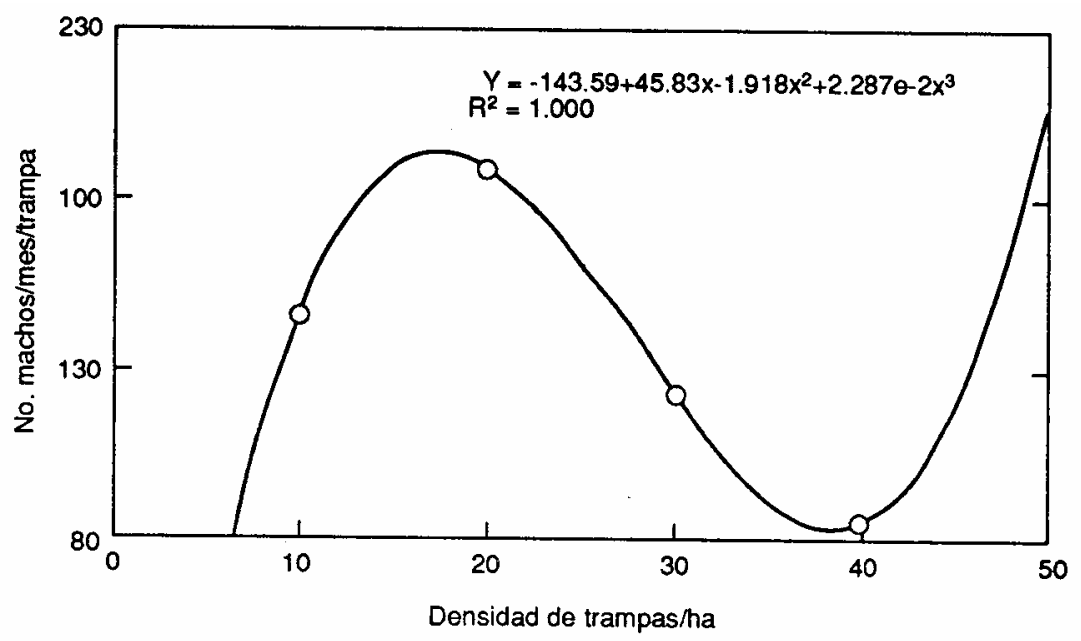

Figura 4. Curva de captura de adultos machos de Tecia solanivora en relación al número de trampas/ha

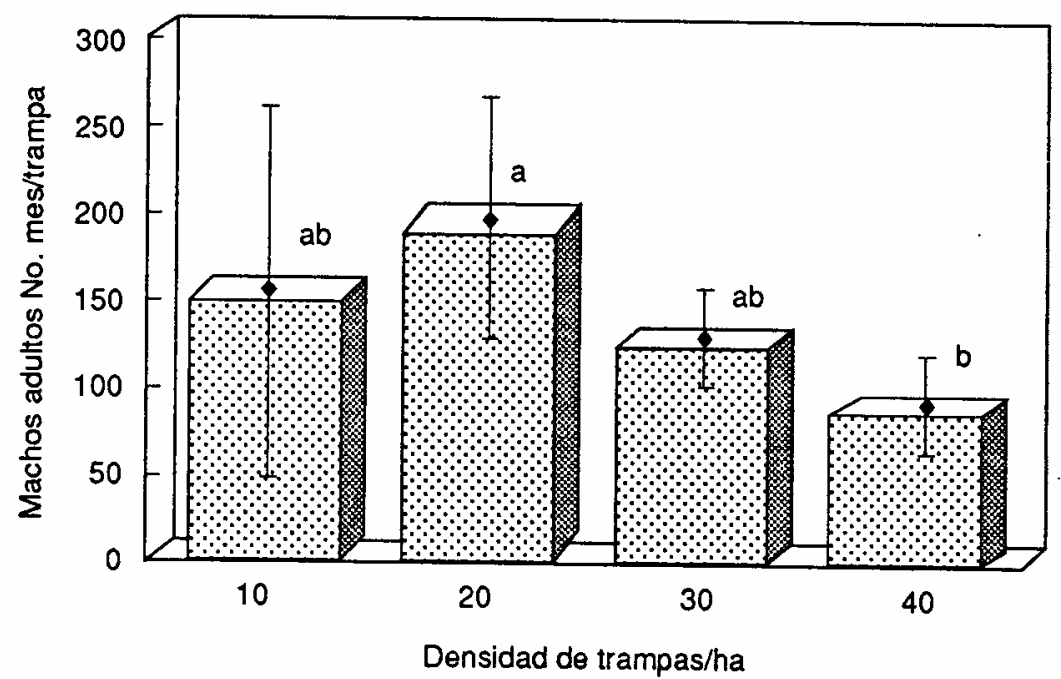

Figura 5. Número de adultos machos de tecia solanivora capturados en trampas de feromona para el lapso junio-septiembre

Valores seguidos por la misma letra, no son significativamente diferentes en la prueba de rango múltiple de Duncan $(\mathrm{p}=0.10)$. 


\section{REFERENCIAS BIBLIOGRÁFICAS}

1. Alvarado, P. J. 1989. Fluctuación poblacional de Scrobipalpopsis solanivora Povolny bajo condiciones de almacén y campo y de Phthorimaea operculella Zeller en campo, en Mucuchies, distrito Rangel, estado Mérida Venezuela En: Ortega Cartaya, E. Manejo Integrado de Polillas de Papa. Memoria IX Reunión Programa Andino Cooperativo de Investigaciones en Papa (PRACIPA). Mar de Plata. Argentina. p. 116-126

2. Alvarado, P. J. 1987. Fluctuación poblacional de Phthorimaea operculella Zeller y Scrobipalpopsis solanivora Povolny bajo condiciones de campo en fincas El Banco y El Baho, estado Mérida, Venezuela. En: Ortega Cartaya, E. Manejo Integrado de Polillas de Papa Memoria IX Reunión Programa Andino Cooperativo de Investigaciones en Papa (PRACIPA). Mar de Plata, Argentina, p. 227-232.

3. Hilje, L.; Cartin, V. 1990. Diagnóstico acerca del combate químico de las polillas de la papa (Lepidoptera: Gelechiidae) en Cartago, Costa Rica. Manejo Integrado de Plagas (Costa Rica) 17:27-33.

4. Leal, H. 1988. La polilla de la papa (Scrobipalpopsis solanivora Povolny) Lepidoptera: Gelechiidae, en Centro América. En: Memoria XXXI Convención Nacional de Entomología. Piura, Perú p. 88.

5. Montero, F.J. 1988. Principales plagas y enfermedades de la papa en Venezuela y su control. En: Ramakrishna, B (ed). Sistemas de Producción en Papa. Manejo de Plagas y Enfermedades. Quito, Ecuador IICABID-PROCIANDINO. p 230-247.

6. Nesbit, B.F.; Bcevor, P.S.; Cork, A.; Holl, D.R.; Murillo, R.M.; Leal, H.R. 1985. Identification of components of the female sex pheromones of the potato tuber moth, Scrobipalpopsis solanivora. Entomol. Expp. Apll. 38:81-85.

7. Ortega, E. 1989. Producción de Semillas de Papa en Venezuela. En: FONAIAP-PRACIPA. Curso sobre Producción de Papa. Barquisimeto, Estación Experimental Lara. p. 1-43.

8. Picón, G. 1989. Metas cumplidas en el área Chama Alta, Mérida, Venezuela. Ministerio de Agricultura y Cría, UEDA. 8 p.

9. Raman, K. V. 1982. Técnicas de confusión de machos para el control de la polilla de la papa. En II Seminario Regional sobre Investigación y combate de las polillas de la papa. Memoria. San José Costa Rica. p. 51-56. 
10. Rincón, J.E; 1987. Manejo Integrado de Polillas de Papa, Caripe. FONAIAP/ PRACIPA. 13p.

11. Rocha, R. M. de la. 1987. Estudio de las feromonas sintéticas de Scrobipalpopsis solanivora y Phthorimaea operculella Zeller para su control. San José, Costa Rica. Ministerio de Agricultura y Ganadería/ PRECODEPA 80p.

12. Rocha, R. M. de la. 1981. La polilla de la papa (Scrobipalpopsis solanivora). Boletín técnico n 69. Guadalupe, Costa Rica. Ministerio de Agricultura y Ganadería. 13p.

13. Rodríguez, C.L.; Lepiz, C. 1990. Muestreo y toma de decisiones para usar insecticidas contra las polillas de la papa. Boletín divulgativo n 94. Ministerio de Agricultura y Ganadería / PRECODEPA 18 p.

14. Rodríguez, C.L.; Lepiz. C. 1988. Manejo adecuado de las feromonas de la polilla de la papa San José, Costa Rica. Ministerio de Agricultura y Ganadería $13 \mathrm{p}$.

15. Salas, J.; Alvarez. C.; Parra. A.; Mendoza, A.O. 1992. Manejo integrado de insectos plagas en el cultivo de la papa en el estado Lara, Venezuela. Barquisimeto FONAIAP / Asociación de Horticultores de Lara / PRACIPA. 66 p.

16. Salazar, J.; Escalante. W. 1984. La polilla guatemalteca de la papa Scrobipalpopsis solanivora, nueva plaga del cultivo de papa en Venezuela. En: Resúmenes XI Jornadas Agronómicas. Maracaibo, Venezuela, p. 24-28.

17. Salazar, J.; Torres. F. 1986. Adaptabilidad y distribución de la polilla guatemalteca de la papa (Scrobipalpopsis solanivora) en el estado Tachira, Venezuela. Agronomía Tropical (Venezuela) 36 (4-6): 137-146.

18. Torres Wills, F. 1990. Principales insectos plagas del cultivo de la papa en Venezuela En: PRACIPA. Taller Aspectos Entomológicos en el Cultivo de la Papa. Memorias. Tibaitata Colombia, p. 46-65.

19. Torres Wills, F. 1989. Algunos aspectos de la biología y comportamiento de la polilla de la papa (Scrobipalpopsis solanivora Povolny) 1973. (Lepidoptera, Gelechiidae) en el estado Tachira (Venezuela). Tesis M. Sc. Facultad de Agronomía. Universidad Central de Venezuela. 86 p. 